\title{
THE STORY OF SILK AND SILKWORM GUT
}

\author{
By A. M. C. Humphries, M.P.S.
}

The silkworm is one of the most astonishing of man's minor conquests. It is as intimately linked with human life as any other living thing we have, and has been so for thousands of years. During all that time it has been born and reared in captivity, a condition made possible because the females do not fly at all, and the males not very well.

This creature, supposed to have originated in the wilds of the Himalayas but first domesticated in China, has less liberty than our poultry; it is as much a creature of the home as a canary, but instead of a cage it has an open tray whereon it passes its larval days, feeding on mulberry leaves. As a moth the female lays 300 or 400 eggs which are hoarded by the silk farmer like precious seed. It may be necessary to keep the eggs in cold storage for a while to prevent hatching whilst the trees are not in leaf; it may be necessary to hasten the hatching.

In the cultivation of silk the moths are forced to lay their eggs on pieces of paper or muslin instead of mulberry leaves, as in their natural state. The eggs are then placed in a room in which the temperature is gradually increased until it reaches $80^{\circ} \mathrm{F}$., and 'in from eight to ten days each egg hatches a small black caterpillar a quarter of an inch long. It is from this point that the feeding, guarding and watching of the crop is done with meticulous care by the farmers or peasants who, for the sake of their pride as well as their pockets, guide the colony to a robust and healthy maturity.

After hatching, the caterpillars are placed on bamboo beds constructed in parallel rows from floor to ceiling of the peasant's house. This stage lasts from six to eight weeks, during which time the worms, fed upon young finely chopped mulberry leaves, cast their skin four times, attaining a length of 2 in. at the final casting.

The caterpillar continues to enjoy its food for a further period of ten days or until it attains its full length of about 3 in. The body of a fullymatured caterpillar consists of 12 segments with six fore-legs and ten hind-legs which are provided with hooks. The mouth is large, with powerful jaws; and the colour is greenish-grey. When many are feeding together the sound of their munching is like that of a breeze in the trees or the murmur of a distant sea. On reaching maturity the caterpillars lose interest in food and are ready to spin cocoons. The peasant accommodatingly provides twigs at the ends of the bamboo beds and the worms innocently creep to them and gather on the leaves; each then sews two leaves together and spins its cocoon between.

The cocoon comprises an outer case of coarse silk, the floss silk of commerce, and within this the caterpillar makes a great winding sheet of pure silk in which to sleep and undergo its transformation.

The cocoon is about the size of a pigeon's egg. The silk is generally yellow but sometimes white; each fibre is double, being spun from a viscid fluid that comes from two tubular glands each connecting with a prominent opening in the lower lip, called a spinneret. The viscous sticky fluies hardens into a strong, glossy thread upon coming into contact with the air. This inner shroud is wrought by spinning together a double line of silk joined to form one line, which reaches a length of from 1,000 to $1,200 \mathrm{ft}$. or more; in fact, $2,400 \mathrm{ft}$. of silk of single line. About 1,600 silkworms are required to produce $\mathrm{I}$ lb. of silk.

Within this cradle the caterpillar casts its skin and becomes a chrysalis, awaiting resurrection into a perfect moth. Such consummation, however, would defeat the entire purpose of the silk farmer, for the moth, to escape from the cocoon, must emit a fluid from her mouth which dissolves the silk at one end and so ruins the whole. It is thus but few for whom there comes an awakening. Only a sufficiency of males and females to hatch out and ensure the next generation are spared. The remainder of the cocoons are submitted to artificial heat which destroys life in the unconscious pupae, and it is human hands and man-made machinery that unwind the lovely silk woven by the worm to be its secret place of retirement during the working of a natural miracle.

The silk which the birth of a moth would spoil is unwound, rewound, washed, dyed and manufactured to deck a queen or a bride, to help a surgeon in his work, and to be the staple of one of the richest commercial enterprises in the world.

What in all nature has a stranger history? Starting from the story of the silkworm in China 
tended for ages by royal hands and kept as a national secret, until a Chinese princess, marrying an Indian prince, smuggled some eggs with her and taught the mystery to the people of India. Thence to the tale of the two Persian monks who, with eggs concealed in hollow canes, came on foot from China to Constantinople, and presented their stolen treasure to the Emperor Justinian, from whose careful treatment of their gift, Europe for I,300 years derived her store of silk and silkworms.

\section{Surgical Silk}

For the manufacture of surgical sutures it is necessary to select a silk of the best quality, having particular regard to evenness in size, cohesion, tensile strength and freedom from foreign matter. When a suitable silk has been decided upon it must be treated in such a manner that the natural gum (sericin) in the silk, while acting as a protection to the fine filaments, will remain flexible. There are many suitable softening agents, but each manufacturer has his own special preference. White oil soap, olive oil and soap, saponified castor oil and a variety of synthetic oils in which organic fats and mineral oils are mixed, have been used. At this stage continued protection of the filaments combined with flexibility is the aim.

The fine ends of silk running about 10,000 yds. to the pound weight are then doubled up, first two at a time, then in multiples, until the desired size is obtained. In the case of Chinese Twist, the silk is twisted by hand first in one direction and then, after three of these singles have been put together, they are twisted again in the opposite direction. The twist is set by one of a variety of methods, such as wet steam, dry steam, damp heat or a combination of vapour, heat and pressure. The Chinese Twist is then finished ready for skeining or spooling, still with its natural silk gum intact.

In the case of plaited silk, the singles are doubled up into the requisite size and a number of singles plaited or braided together. The least number is four and it is unusual to build machinery to plait together more than 24 singles. Either before or after the plaiting process, the natural gum, is removed by the simple process of boiling in a solution of white oil soap. At every stage of manufacture the emphasis must be on the maintenance of exact diameter sizes and tensile strength. This can only be done by building up sizes step by step and checking at every stage.

For surgical use, silk is sterilized by boiling or autoclaving.

\section{Silkworm Gut}

In the preparation of silkworm gut the cater- pillars on reaching maturity are not allowed to spin their cocoons. The peasant, who has s tenderly cared for his charges up to this pointe suddenly turns traitor and rudely dumps then: into a vinegar bath, putting him within reach of his goal but putting the caterpillars for ever beyon $\Phi$ reach of theirs. The viscous sticky fluid, whict would harden upon coming into contact with ai into the strong glossy thread of silk, is prevente $\bar{\phi}$ by the vinegar bath from being spun into a cocoon and a peasant girl with deft fingers extracts the tubes and lays them across her knees, stretching them to their full length.

Silkworm gut, even to this day, is almos exclusively produced in the location where it has its inception some 60 or 70 years ago-the city of Murcia, capital of a sunny province of the sami name in south-easterly Spain. Although manK other localities have attempted the production $0_{0}^{\circ}$ silkworm gut, the climate and soil of Murcia have given the populace of the community a virtualo monopoly of the product. For miles around the city itself, the so-called 'Huerta' peasants may beseen cultivating the silkworm for its silk or fo gut, the proportion of each depending upon thछ rise and fall of the market.

There is a common fallacy that the size of gut can be controlled by the operator, as it appearsoto stand to reason that, if heavy gut is required, is iso only necessary to draw the sac to a short lengthp whilst, if fine gut is desired, it can be obtained bys pulling the sac to whatever length may be neces $\frac{\supset}{0}$ sary. This is not the case. A sac can only be drawn to a certain length. If too long or too shortळ the result is a spoilt piece of gut; everything depends upon the judgment of the operator. Fof this reason, the manufacturer has no more controb over the sizes and lengths of silkworm gut than he would have over a crop of potatoes, and this is the explanation of the fact that it is impossible for any but the largest manufacturers to guarantee to supply the exact lengths ordered.

When his entire crop is dry, the peasant ties the material into bundles and carries them to market where they are sold according to weight. Ino appearance these bundles are like hay, the gut being covered with a thin yellow coating known ass 'carne' and, because sizes, lengths and qualities are mixed in apparently inextricable confusiono great experience and judgment are required on the part of buyers in estimating the quality and value of packages of raw gut.

The material having reached what the silkworm gut manufacturer recognises as the first raw state it is now ready to go through the various stages of manufacture. The raw gut is usually bought in the 'Huerta' in lots of 10 to 20 bundles, each 5 bundle weighing approximately I lb. It is brought居 
to the factory, weighed and paid for accordingly. The gut is then classified roughly into three thicknesses - thin, medium and thick-and afterwards tied together with strings in bundles of approximately $\mathrm{I} \mathrm{lb}$. It is now ready for boiling.

The gut is immersed and boiled in a kettle of soapy water, each kettle containing about $30 \mathrm{lb}$. It remains in the kettle about 20 minutes or long enough to dissolve the yellow coating around it. As soon as the gut is taken out of the boiling water, it is washed, bundle by bundle, in fresh cold water, after which the gut is hung in the bright sun to be bleached. If the weather should be unfavourable or the sky cloudy, it is better not to dry the gut, for it would not then develop the strong brilliancy which the sun produces. The best time of the year to boil and dry the gut therefore is during the summer.

Selectors. After bleaching, each pound bundle of gut is separated into two or three smaller bundles and, aftering being moistened in water for half an hour, these bundles are handed over to the selectors, who classify the gut strand by strand, according to thickness:

THIN is carefully assorted into four thicknesses -Refina, Fina, Regular and Padron 2.

Medium is assorted into Reguilar, Padron 2, Padron I, Marana 2 and Marana $\mathrm{I}$.

THICK-The thickest bundles are now classified into Padron I, Marana 2, Marana I, Imperial and Hebra.

After this sorting, all the gut is classified once more by highly trained workers. The same gut passes five or six times through the hands of the selecting girls, and forewomen are constantly checking the work with steel gauges. Furthermore, in order that the brilliancy and the strength of the gut may be enhanced, it is again exposed to the brilliant Spanish sun for about six hours each day.

Measurers. As soon as the selecting girls have classified some 50 to $100 \mathrm{lb}$. of each quality, the measuring girls start their work and measure thickness by thickness, starting for example with Padron 2. When this grade is finished they go on to Padron I, and so on. There must always be a stock of about $300 \mathrm{lb}$., so that the measuring girls have five or six days' work in the same thickness. The girls moisten the gut in water before starting to measure strand by strand and proceed in the following manner:

Each takes a handful of gut (about $4 \mathrm{oz}$.), moistens it, and envelops it firmly in a linen cloth in order to make the gut more flexible. For the measurement of the gut they avail themselves of an instrument made of tin, called ' lata' in Spanish. It contains 24 measurements from 7 in. to $18 \frac{1}{2}$ in. The gut is placed, strand by strand, in the space provided in the tin container. During five hours of the day these girls measure gut, whilst the $\stackrel{\otimes}{\circ}$ remaining three hours of their working time they $c$ devote to taking out the gut from the measuring $\Rightarrow$ instrument, and arranging it length by length until $\stackrel{\text { o }}{+}$ the instrument is empty. The arrangement of the gut consists of evening the strands so that all the 흠 curly ends ('grilla') of both sides are together, $\frac{\bar{D}}{\square}$ and to avoid having any strand protruding. The $\propto$ gut is then tied at both ends with a thin string and $\infty$ is carefully dried by applying a piece of chamois $\vec{\circ}$ and rubbing it up and down, thus leaving the gut thoroughly dry, strong and white.

Counting girls and classifiers of quality. When the measuring girls have measured a considerable $\stackrel{0}{0}$ quantity, say, roo to $200 \mathrm{lb}$. or approximately 300,000 to 600,000 strands, of Padron 2 or Padron W $^{2}$ I, or of any other thickness, the counting girls $N$ start their work. The gut is moistened once $\infty$ more at both ends, without touching the good ${ }_{\infty}$ centre part of the strand. Through this moistening $\omega$ they are better able to separate the strands and $\frac{\text { o }}{2}$ avoid having ends tear when they are separated $\vec{O}$ entirely. If these ends were not moistened, the $O$ gut might suffer damage, for the curly ends $\stackrel{\circ}{\circ}$ necessary for the handling during the elaboration $\stackrel{\Phi}{-}$ of the gut would break off. The strands are $\overrightarrow{0}$ classified into the following five qualities:

(a) Natural Selecta. This is perfect gut and requires no improvement.

(b) SEL ECTA (for mazantining purposes). Good quality gut which can be improved by mazantining.

(c) NATURAL SUPERIOR. Good second quality $\stackrel{\circ}{\stackrel{2}{\varrho}}$ which cannot be improved.

(d) SUPERIOR (for mazantining purposes). 윽 Second quality which can be imprcved by mazantining.

(e) Estriada. Third quality.

Once the gut is classified in the above manner the strands are counted into hanks of ro4 strands.

Pressers. The pressing or stretching of the strands is done by strong men, using presses or $\delta$ sets of vices. The pressing of the gut is a pre- $₹$ liminary work necessary for the process of mazan- 은 tining the gut, for if the strands were not well $D$ stretched the labour of the mazantinera would be twice as difficult. Each press consists of two iron vices attached to a wall in opposed vertical o positions. The worker takes about Io or $12 \tilde{O}$ hanks of 100 strands each, arranges them flat in his N hand, covers the upper ends of the strands with a piece of leather for protection against any possible damage by the vice, and then places them flat in the upper vice, screwing it tightly together. The $\stackrel{?}{?}$ upper vice, which can be screwed upwards and 7 downwards, is then lowered until the other end of the strands of gut reach the lower vice. Her 
too, the hanks are arranged flat, then likewise protected by another piece of leather and placed in the lower vice, which is then closed. Now the upper vice is screwed gradually upwards, thereby creating a tension, and once more both vices are screwed more tightly until the strands of gut remain thoroughly taut between them. The worker then takes an iron clamp, the middle of which is lined with chamois, places the iron around the gut suspended between the two vices and commences a fijctioning movement up and down the gut. The strenuous work of this friction applied to the gut leaves the strands, after about I 5 to 20 up-and-down strokes, well stretched and evened.

Mazantining. This name is applied to the process of polishing for the improvement of the quality of the gut. The mazantinera must work in good daylight. She sits on a low stool, having before her a wooden stand to which a small vice is attached in an inclined position. The mazantinera takes a hank of 100 strands, separates it into three parts and starts her work by putting one end of it into the vice. She moistens the defective part of the gut with water by applying a wet cloth. Then she takes a dry linen cloth and strongly rubs it against the part of the gut that has been moistened and, through the strong friction and pressure of her fingers, removes the slight defects of the gut. She applies this process to those parts of the gut which her eye detects need improvement. By the process of rubbing, very fine particles of the gut are removed, thus slightly thinning the material. The appearance of the gut through this process is noticeably improved.

Girls counting mazantined gut. In the process of pressing and mazantining the gut some strands are broken. It is therefore necessary to recount the material. Furthermore, the thickness of the gut may have been altered; reclassification is therefore required.

Tyers. These girls tie each 104 strands at both ends with a blue or red string, according to the thickness. Measurement of the gut is taken from grilla to grilla (from curly end to curly end).

Wrapping gut. Before wrapping, men measure the gut once more, hank by hank, thus checking the work of the previous group, and tie together Io hanks into bundles of I,000 strands each, and again into bundles of 2,000 to 8,000 strands. In order to protect the gut, the bundles are wrapped entirely in blue paper, not, however, befcre it is once more inspected by an expert.

It takes from four to six weeks to convert the raw gut into the finished product. As will be apparent from the above description the manufacture of silkworm gut is so dependent upon the human element that it cannot be reduced to mathematical or scientific precision. At no stage of the process so far has it been possible to replaç human labour by a machine. It follows that the consumers of this important article are largel dependent upon the skill of the individuaf manufacturers.

\section{Gut Drawing}

So far we have dealt with the various stages through which the natural gut passes from the time it leaves the hands of the Spanish peasant. until it reaches its finished state, but there are other stages of manufacture through which a con $\overrightarrow{\vec{\omega}}$ siderable proportion of the natural gut has yẹ to pass. The most important of these is that diawing.

It might be supposed that having so far handled every stage in the manufacture of the natural gutin the Spanish workers would be quite competent tọ carry out this delicate work. Actual experience however, has proved that the average Spanish girt is not sufficiently painstaking to be entrusted witi the manufacture of the gut beyond this stage. It is therefore now shipped to this country.

Up to this stage, the classification of the guô has been only rough. It has therefore to b@ repeated before commencing the process of draw= ing, which is such a delicate operation that ogly the very best gut can be used for the purpose. The Selecta or finest quality gut, therefore, is omcE more picked by highly-trained English workers a Redditch.

Apart from the three qualities into which the gut has been selected in the Spanish factory, has also been sorted into the following thicknesses + t

\begin{tabular}{|c|c|c|c|c|}
\hline \multicolumn{5}{|c|}{ Thickness in 1,000 th of an inch } \\
\hline Hebıa .. & . & - & . & 20 and over \\
\hline Imperial & .. & .. & $\ldots$ & I 8 to 20 \\
\hline Marana I & . & $\cdots$ & $\cdots$ & 16 to 18 \\
\hline Marana 2 & .. & . & . & 15 to 17 \\
\hline Padion I & .. & . & . & 14 to 16 \\
\hline Padron 2 & . & . & . & I3 to 15 \\
\hline Regular & .. & . & .. & 12 to 14 \\
\hline Fina $\quad \ldots$ & . & . & . & I I to 13 \\
\hline Refina & . & . & . & 9 to 12 \\
\hline
\end{tabular}

Thus it will be seen that every conceivable thickness of strand produced, from the finest t $\overline{\bar{\Omega}}$ the stoutest, is sorted into nine different grades? It is natural, thereforè, that there should be conf siderable overlapping in the thicknesses, and that strands should be found in one grade which appeaefiner or stouter than others in the same grade This effect is still more accentuated by the facf that a large proportion of the strands, even in the Selecta gut, will taper to a greater or lesser degreet from the centre to the end of the strand. Whils $\overline{0}$ these tapering strands may be perfectly good gut 
in their present state they are not desirable for surgical gut since, for this purpose, the strands must be as level as possible. This brings us to one of the reasons for gut drawing, by which the strands are reduced to a perfectly level thickness throughout their length.

But another and perhaps more important reason for the drawing of gut is the fact that natural gut is rarely produced finer than $9 / 1,000$ ths in. in thickness through its strands, while for many surgical operations finer gut than this is required. Thus Refina gut, which may average perhaps ro/ 1,000 ths in. in thickness, may in places only measure 7 or $8 / 1,000$ ths in. and in other parts perhaps II/ 1,000 ths in. Such a strand can be drawn down to $5 \mathrm{x}$, which pnly measures $6 / 1,000$ ths in. in thickness, without losing much of its strength. The process of drawing therefore gives the gut a perfectly level and even surface throughout the strand, which is of comparatively great strength.

Before the gut can be drawn the ends of the strands have to be pointed. This is very hard and tedious work, since each strand has to be literally sharpened like the end of a pencil. To do this, the worker takes a few strands at a time, holding the ends on a stone slab, at the same time rubbing them very vigorously in an outward direction with a chamois leather on which a mixture of water and bath-brick has been smeared. As will be supposed, it takes a considerable time to complete the pointing of the gut, but once this work is well done, the actual drawing occupies comparatively little time.

Having been pointed, the strands are now ready to pass through the plate. This is a metal plate measuring approximately $3 \frac{1}{2}$ in. by $\mathrm{I}$ in., on which is mounted a number of jewels, each bored with minute round holes of the desired size. The pointed end of the gut is now passed through the appropriate hole, according to the thickness of the gut required, and pulled through with small metal pliers. By this means the outer coating of the gut is shaved off, leaving the inner core perfectly round and level in thickness. After drawing, the gut is stained to the required colour and strenuously polished in a manner very similar to the process of mazantining already described. Even now, however, we have not finished with the selection of the gut, since it has yet to be picked once more for quality. Great skill is required in the drawing of the gut, as a good drawer will obtain a far greater percentage of best gut than an unskilled worker.

A very important factor in producing the best drawn gut is the choice of the correct grades of natural gut. If the average thickness of the gut to be used for drawing is more than $3 / 1,000$ ths in. above that of the drawn gut it is desired to produce, the gut will have to be passed through seyeral holes in the plate. Thus the gut drawer has to use considerable judgment as to whether it is desired to draw the gut through more than one hole and, in the case of drawing the very fine grades, it is always necessary to commence with a mediumsized hole such as $3 \mathrm{X}$ and pass the gut through each succeeding hole until the desired thickness has been reached. If this is not done a broken strand will result. It is natural that a certain number of strands will break in the plate but, here again, a skilled worker will break far less strands in the drawing than one who is inexperienced.

In view of the tremendous amount of detail and tedious. work involved in the production of one strand of gut, it is amazing that it can be produced at so low a price.

For surgical use, silkworm gut is sterilized by boiling.

\section{Japanese Gut}

In view of the popularity of silkworm gut, it is not surprising that practically every silk-producing country in the world should have attempted its production. It is a remarkable fact that no other country has met with success. Italy, Turkey, Bulgaria, Greece, Egypt, India, Japan and China have all endeavoured at various times to produces silkworm gut, but each has met with utter failure.? Italy is a possible exception, but even this country, which is responsible for 75 per cent. of the European production of silk, has produced negligible quantities of silkworm gut, and the Italians have become more or less resigned to their inability to produce it. The Japanese, however, were not so easily discouraged and, whilst they are still unable to manufacture the actual article, they have succeeded in producing a very useful substitute.

The manufacture of fapanese gut is conducted on totally different lines from those of the Spanish manufacturers. Instead of extracting the glands from the silkworm, it is allowed to spin its cocoon. The silk is then wound to yarn of the required thickness, after which it is slowly passed through a boiling preparation of fine grade animal glue and an extract from seaweed. In the process of boiling, the silk being itself an animal substance, it is reduced almost to a semi-liquid state, so that it becomes saturated with the properties of the glue-like substance in which it is boiled.

When the material is drawn off it is very sticky and, as it is important that the finished product should be hard and clear, special chemicals have to be used to dry it quickly in order to avoid the adher ence of dust or moisture. 
One of the most important factors in the manufacture of Japanese gut is the proportion of silk to be used. In order to obtain the maximum strength and elasticity it is necessary to use the greatest possible proportion of pure silk, but at the same time a proportion of glue-like substance is necessary if the finished product is to resemble silkworm gut. By using a large percentage of glue, Japanese gut can be made to look practically identical with the genuine article, but this cannot be done without considerably sacrificing its strength. Conse- sequently a compromise has been reached, and the most satisfactory ratio has been found to be $8 \frac{1}{2}$ per cent. pure silk and 15 per cent. glue. Even with these proportions, however, the silk does net: take on so glossy an appearance as one would wish so that to the uninitiated it may appear to be less desirable than the shiny production.

Since the war Japanese gut has not yet bee available in any quantity in this country. $\subseteq$

For surgical use, Japanese synthetic gut sterilized by boiling.

\title{
BOOKS RECEIVED
}

\begin{abstract}
The Editorial Board acknowledge with thanks the receipt of the following volumes. $A$ selection from these will be made for review.
\end{abstract}

'Essentials of Orthopaedics.' By Philip Wiles, M.S., F.R.C.S., F.A.C.S. Pp. xv +486 , with 7 colour plates and 365 text figures. London: J. \& A. Churchill Ltd. r949. 42 .

'A Surgeons Guide to Local Anaesthesia.' By C. E. Corlette, M.D., Ch.M., F.R.A.C.S. Pp. xi + 355, with 200 illustrations. Bristol: John Wright \& Sons. London: Simpkin Marshall Ltd. I 948 . 35 s.

'Blood Transfusion.' Edited by Geoffrey Keynes, M.A., M.D., F.R.C.S. Pp. xii + 574, with I Io illustrations. Bristol: John Wright \& Sons. r949. 52s. 6d.

'Gas and Air Analgesia.' By R. F. Minnitt, M.D., D.A., F.F.A., R.C.S. 4th Edition. Pp. vii +86 , with 22 illustrations. London: Baillière, Tindall \& Cox. r 949.5 s.

'Psychological Aspects of Clinical Medicine.' By Stephen Barton Hall, M.D., D.P.M. Pp. xii +416 , with 6 illustrations. London: H. K. Lewis \& Co. I949. 21S.

'Symptoms in Diagnosis.' By J. C. Meakins, C.B.E., M.D., D.Sc. 2nd Edition. Pp. xv + 542, with 112 illustrations. London: Baillière, Tindall \& Cox. r949. 42s.

'An Account of Schools of Surgery.' By T. D. H. Widdess, M.A., L.R.C.P., F.C.S.I. Pp. 107, with 15 plates. Edinburgh: E. \& S. Livingstone. 1949. I7s. 6d.

'Cystoscopy and Urography.' By F. B. Macalpine, D.Sc., F.R.C.S. 3rd Edition. Pp. 570, with 338 illustrations and 15 colour plates. Bristol : John Wright \& Sons, Ltd. I $949.63 s$.

'Recent Advances in Oto-Laryngology.' By R. Scott Stevenson, M.D., Ch.B., F.R.C.S.(Ed.). 2nd Edition. Pp. 395, with 106 illustrations and 8 plates. London: J. \& A. Churchill. r949. 24s.

'The Science and Art of Joint Manipulation.' Vol. I. By James Mennell, M.A., M.D., B.C. and Edition. Pp. 215, with 299 illustrations. London: J. \& A. Churchill. 1949. 24 s.
' Sir William Gowers, $1845-191$ 5.' By Macdona两 Critchley. Pp. I 8 , with ro plates. London: William Heinemann. 1949. 17s. 6d.

'Modern Treatment Year Book, I 949.' Edite्g by Sir Cecil Wakeley, K.B.E., D.Sc., F.R.C.So, F.R.S.E. Pp. viii + 338, with 39 illustrations. London: Medical Press. 1949. I 5 s.

'Sternal Puncture.' By A. Piney, Msto M.R.C.P., and J. I. Hamilton-Paterson, MĒD. F.R.C.S. 4th Edition. Pp. 89, with I4 plates. London: William Heinemann. I 949. I 5 s.

'A Companion in Surgical Studies.' By I Aird, Ch.M., F.R.C.S. Pp. viii + ro6o. Edir burgh: E. \& S. Livingstone. 1949. 63s.

'Illustrations of Surgical Treatment.' By Erif L. Farquaharson, M.D., F.R.C.S. 3rd Fditioß Pp. 39I, with 6I plates. Edinburgh: E. \& \$. Livingstone. 1949. 25s.

'A History of Oto-Laryngology.' By Scoit Stevenson, M.D., F.R.C.S., and Douglas Guthries M.D., F.R.C.S. Pp. vii + 155, with 52 plate Edinburgh: E. \& S. Livingstone. r 949. I7s. 6क. 'Mollison's Forensic Medicine Lectures.' Bgy. K. M. Bowden, M.S. 5th Edition. Pp. 282, witg 65 figures. Melbourne: W. Ramsey (Surg.) Ptys, Ltd. I 949 . 37s. 6d.

'Atlas of Plastic Surgery.' By M. I. Bersō M.D., Pp. xii +304 , with 1,200 illustrationg? London: William Heinemann Medical Books Lt黑 1948. 75s.

'The Mental and Physical Effects of Pain.' BY V. C. Medvei, M.D., M.R.C.P. Pp. 59. Edin burgh: E. \& S. Livingstone Ltd. 1949. 3 s.

'The Rhesus Factor.' By G. Fulton Roberts M.A., M.B., M.R.C.S., L.R.C.P. 2nd Edition. Pp. 64. London: William Heinemann Medica Books Ltd. 1949. 3s. 6d.

'A Practice of Orthopaedic Surgery.' By T. P. McMurray, C.B.E., M.B., B.Ch., F.R.C.S. 3rid Edition. Pp. viii +444 , with I9I illustration? London: Edward Arnold \& Co. 1949. 30 . 